\title{
Long swings in exchange rates: a stochastic control approach
}

\author{
Rosella Castellano ${ }^{\mathrm{a}}$ and Rita L. D’Ecclesia ${ }^{\mathrm{b}}$ \\ ${ }^{a}$ Dipartimento di Istituzioni Economiche e Finanziarie, University of Macerata, Via Crescimbeni n. 20, 62100 Macerata, \\ Italy, \\ ${ }^{\mathrm{b}}$ Dipartimento di Teoria Economica e Metodi Quantitativi per le Scelte Politiche, University of Rome "La Sapienza", \\ P.le Aldo Moro n. 5, 00185 Roma, Italy \\ E-mails: castellano@unimc.it [Castellano],rita.decclesia@uniroma1.it [D’Ecclesia]
}

Received 4 October 2005; received in revised form 25 January 2007; accepted 14 April 2007

\begin{abstract}
A regime-switching model to describe the exchange rate dynamics is derived as solution to a stochastic control problem. We assume exchange rates evolve according to some macroeconomic variables (fundamental) whose dynamics could be described by a Brownian motion with a state-dependent drift. The local Monetary Authority is assumed to intervene influencing the evolution of the fundamental, causing the exchange rate to switch from a depreciating to an appreciating regime (and vice versa). We assume the behaviour of the Monetary Authority can be modeled using an optimal control framework where the state variable is represented by the fundamental. The solution of the model allows the determination of an endogenous tolerance band within which the exchange rate freely fluctuates.
\end{abstract}

Keywords: long swings; stochastic control; monetary authorities' intervention; time-consistent policy

\section{Introduction}

Modeling exchange rate dynamics has been a major goal of scholars and practitioners. Two main approaches have been used in the recent literature to study exchange rate dynamics: the Markov Switching Model (MSM) and the Random Walk Hypothesis (RWH).

The power of the MSM for exchange rate modeling was first demonstrated by Engel and Hamilton (1990) and Engel (1994), who were able to identify the presence of long swings in exchange rates. Using an econometric approach, they decompose a non-stationary time series into a sequence of stochastic segmented time trends with two regimes (states). The mean of the exchange rate changes may vary across regimes allowing the identification of long swings.

Evidence of long swings behavior in exchange rates have also been reported in Evans and Lewis (1995), Marsh (2000), Bollen et al. (2000), Dewachter (2001) and Clarida et al. (2003), 
showing that MSM is able to describe the exchange rate dynamics, but it is unstable over time and not suitable for forecasting.

The RWH was first introduced by Meese and Rogoff (1983) evidencing how the random walk forecasts outperform the results obtained using structural exchange rate models. More recent studies by Meese and Rose (1991), MacDonald and Taylor (1994), Chinn and Meese (1995), MacDonald (1999) and Cheung et al. (2005) and Cheung and Erlandsson (2005) have also reported the inadequacy of traditional exchange rate models, structural and time-series models, to provide accurate forecasts of exchange rates. However, the RWH remains unsatisfactory, from an economic point of view, as it ignores the impact of the fundamental.

We believe that 'long swings in the exchange rate pose important challenges for existing theory' (Engel and Hamilton, 1990, p. 689), hence the presence of a long-swinging behavior has important implications for modeling exchange rate dynamics and forces the investigation of what factors may lead to such switching dynamics. Kaminsky (1993), for example, shows that a move from a recessive to an expansionary monetary policy increases the exchange rate depreciation resulting in long swings.

Evans and Lewis (1995) assume that exchange rate switches between appreciating and depreciating regimes are incorporated into rational traders' forecasts of the future exchange rate. They use the monetary model and assume that the fundamental switches between a depreciating regime and an appreciating one. They also introduce jumps occurring every time a switch of the fundamental occurs; hence, exchange rate dynamics are described by a sequence of mixed jump processes.

The aim of this paper is to capture the swinging behavior of the exchange rate and to provide an economic explanation for it. Exchange rate is too important to be left to the market and hence Monetary Authorities (MMAA) have frequently, and at times, forcefully, intervened using monetary policy instruments to influence the path of their respective currencies. In this context, MMAA's interventions are required to keep exchange rates in line with their long-run fundamental value. The fact that most Central Banks attempt to manage their exchange rates stresses an important question, i.e. 'what role can they play in achieving the desired exchange rate goal?'.

We assume that domestic MMAA may control the evolution of the fundamental; hence, exchange rate dynamics could be better described by a managed floating regime. This assumption allows the use of a stochastic optimal control approach to model MMAA's interventions.

Given that in the long run the fundamental matters (MacDonald, 1999; Mark, 1995), its dynamics could be described by a stochastic differential equation. Following Krugman (1991, 1992), we assume that the exchange rate is a nonlinear function of the fundamental and MMAA want to keep it not too far from its long-run equilibrium level. This may result in changes of monetary policies, which may cause a change of the instantaneous drift of the fundamental bringing it closer to its equilibrium level and, at the same time, producing a switch in regime: from a depreciating one (regime 1) to an appreciating one (regime 2), and vice versa. The switching points are optimally determined as solution of the stochastic control problem. We also contribute to the re-examination of the monetary model in a time-varying coefficients context, showing that regime-switching properties provide substantial explanatory power.

A first validation of the proposed model is obtained using the monthly D-Mark/US-dollar exchange rate over the period 1973-2005. Evidence of regime switches for D-Mark/US-dollar is provided. 
The paper is structured as follows: Section 2 presents the stochastic control approach and its solution; Section 3 reports the estimation of the model based on the Method of Simulated Moments and the statistics for the estimated parameters; Section 4 provides some concluding remarks.

\section{The theoretical model}

We assume the exchange rate is a nonlinear function of the fundamental whose dynamics are determined by a set of macroeconomic variables and policy instruments. MMAA set a long-run equilibrium level for the fundamental. They monitor the dynamics of the current fundamental in order to avoid its 'undesired' changes.

In this context, we define an optimal control model that describes MMAA's behavior. The control variable is represented by the drift of the process used to model the fundamental's dynamics.

\subsection{The exchange rate}

According to the standard flexible price monetary model, the spot exchange rate at time $t$ is described by

$$
s_{t} \mathrm{~d} t=f_{t} \mathrm{~d} t+\lambda E_{t}\left[\mathrm{~d} s_{t}\right], \quad \lambda>0
$$

where $s_{t}$ is the logarithm of the exchange rate defined as unit of domestic currency per unit of the reference currency; $f_{t}$ denotes the logarithm of a set of macroeconomic variables (fundamental) driven by a Brownian motion with constant instantaneous variance, $\sigma^{2} ; \lambda$ can be interpreted as the semi-elasticity of domestic money demand with respect to the interest rate and $E_{t}\left[\mathrm{~d} s_{t}\right]$ measures the expected change of the exchange rate at time $t$. Both $s_{t}$ and $f_{t}$ are assumed to be observable at time $t$ (Svensson, 1992).

The fundamental, $f_{t}$, can be decomposed in an endogenous component, sensitive to MMAA's intervention, and an exogenous one. The process for the fundamental, $f_{t}$, is described by the stochastic differential equation (SDE)

$$
\mathrm{d} f_{t}=\theta\left(f_{t}\right) \mathrm{d} t+\sigma \mathrm{d} B_{t},
$$

where $\theta\left(f_{t}\right)$ is the instantaneous drift, $\sigma^{2}>0$ is the instantaneous variance and $B_{t}$ is a standard Brownian motion.

The theoretical fundamental, which represents the long-run equilibrium level, depends on a set of macroeconomic variables that affect the exchange rate. We assume its changes can be described by

$$
\mathrm{d} \tilde{f}_{t}=\beta_{t} \mathrm{~d} t, \quad \beta_{t}: \Re \rightarrow \Re .
$$

We suppose that MMAA intervene in order to maintain the fundamental, $f_{t}$, broadly in line with the theoretical value, $\tilde{f}_{t}$. Doing so, we may assume MMAA's aim is to minimize the difference $x_{t}=f_{t}-\tilde{f}_{t}$. 
Interventions are justified by Mussa (1981): 'A valid case for interventions lies on the ground that the authorities may have better knowledge of their own future policy intentions than private market participants'. MMAA can use standard monetary policy tools to modify the fundamental's dynamics. Several approaches describe the effects of these interventions on the dynamics of the exchange rates (among others, Miller and Zhang, 1996; Mundaca, 2001).

In this work we assume that MMAA intervene to modify the rate of instantaneous change of the fundamental, $\theta\left(f_{t}\right)$, representing the control variable.

Formally, the dynamics of $x_{t}$ on $\left(\Omega, \Im, P^{\bar{x}}\right)$ is given by

$$
\mathrm{d} x_{t}=\mathrm{d} f_{t}-\mathrm{d} \tilde{f}_{t}=\left[\theta\left(f_{t}\right)-\beta_{t}\right] \mathrm{d} t+\sigma \mathrm{d} B_{t},
$$

where $\theta\left(f_{t}\right)$ and $\beta_{t}$ are bounded functions, and $P^{\bar{x}}$ defines the probability measure of a Brownian motion with initial position $\bar{x}=x_{0} \in \Re$. Applying stochastic calculus, ${ }^{1}$ we have

$$
\mathrm{d} x_{t}=\theta\left(f_{t}\right) \mathrm{d} t+\sigma \mathrm{d} \hat{B}_{t} \quad \text { with } \mathrm{d} \hat{B}_{t}=\mathrm{d} B_{t}-\frac{\beta_{t}}{\sigma} \mathrm{d} t
$$

where $\hat{\beta}_{t}$ is defined on $(\Omega, \Im, \hat{P})$. The equivalent probability measure $\hat{P}$ is related to $P$ by

$$
\left.\frac{\mathrm{d} \hat{P}}{\mathrm{~d} P}\right|_{\Im_{t}}=\exp \left[\int_{0}^{t} \phi_{s} \mathrm{~d} B_{s}-\frac{1}{2} \int_{0}^{t} \phi_{s}^{2} \mathrm{~d} s\right] .
$$

According to (5) the process, $x_{t}$, has the same drift, $\theta\left(f_{t}\right)$, of the fundamental, $f_{t}$, and the Monetary Authority's goal is realized minimizing the expected discounted quadratic deviation of $x_{t}$ from the origin

$$
J(\bar{x}, \theta)=\min _{\theta \in \Theta} \hat{E}^{\bar{x}}\left\{e^{-\gamma t} \int_{0}^{\infty} x_{t}^{2} \mathrm{~d} t\right\} \text { for } \bar{x}=x_{0} .
$$

The minimization is performed setting the optimal drift $\theta^{*}\left(f_{t}\right) \in \Theta$, with $\Theta=$ set of Borel measurable functions; $\bar{x}=x_{0}=f_{0}-\tilde{f}_{0}$ is the initial value of $x_{i} ; \gamma$ is the discounting factor $(\gamma>0)$; $\hat{E}^{\bar{x}}$ is the expected value corresponding to a Brownian motion with initial position $\bar{x} \in \Re$.

In terms of optimal stochastic control the problem becomes

$$
\begin{aligned}
& J\left(\bar{x}, \theta^{*}\right)=\min _{\theta \in \Theta} J(\bar{x}, \theta), \quad \forall \bar{x} \in \Re, \\
& \text { s.t. } \mathrm{d} x_{t}=\theta\left(f_{t}\right) \mathrm{d} t+\sigma \mathrm{d} \hat{B}_{t} .
\end{aligned}
$$

In (5), we assume $\theta\left(f_{t}\right)$ absolutely continuous with a rate of change bounded between $\theta_{1}$ and $\theta_{2}$ : $\theta_{1}<\theta\left(f_{t}\right)<\theta_{2} ;\left[\theta_{1}, \theta_{2}\right]$ represent the extreme sustainable measures to curb the dynamics of the fundamental and the drift $\theta\left(f_{t}\right)$ is equal to $\theta_{1}, \theta_{1}<0$ when contractionary monetary policies are introduced and it is equal to $\theta_{2}, \theta_{2}>0$ when expansionary monetary policies are adopted.

\footnotetext{
${ }^{1}$ According to Girsanov's Theorem it is possible to estimate the probability measure of the new Brownian motion, $\hat{B}_{t}$. 


\subsection{The solution of the stochastic control problem}

The formal Hamilton-Jacobi-Bellman equation to solve the control problem (8) is

$$
\gamma J=x^{2}+\frac{\sigma^{2}}{2} J_{x x}+\inf _{\theta_{1}<\theta\left(f_{t}\right)<\theta_{2}}\left\{\theta\left(f_{t}\right) J_{x}\right\},
$$

where subscripts identify derivatives.

We expect a solution based on the sign of $J_{x}$ and we look for two numbers $\delta_{i}\left(\theta_{j}, \gamma\right)$, for $i=1,2$; $j=0,1,2$, with $\delta_{1}(\cdot)>0$ and $\delta_{2}(\cdot)<0$, identifying the switching points of the process $x_{t}$. More precisely, $\delta_{1}(\cdot)$ and $\delta_{2}(\cdot)$ represent the upper and lower threshold values that define an endogenous time-varying 'tolerance band' and a time-consistent policy. We assume that if $x_{t}$ exceeds the threshold values, $\delta_{i}(\cdot)(i=1,2)$, MMAA's interventions occur:

- if $x_{t} \geqslant \delta_{1}(\cdot)>0$ restrictive monetary policies are adopted causing the fundamental to switch from an appreciating regime to a depreciating one;

- if $x_{t} \geqslant \delta_{2}(\cdot)<0$ expansionary policies are introduced causing the switch from the depreciating regime to the appreciating one;

- if $\delta_{2}(\cdot)<x_{t}<\delta_{1}(\cdot)$ no interventions occur and the fundamental follows the dynamics described by equation (2).

According to the initial position of $x, \bar{x}=x_{0}$, the corresponding formalization of (9) is

(1) $\gamma J=x^{2}+\frac{\sigma^{2}}{2} J_{x x}+\theta_{2} J_{x}, \quad J_{x} \leqslant 0, \quad \bar{x} \leqslant \delta_{2}(\cdot)$,

(2) $\gamma J=x^{2}+\frac{\sigma^{2}}{2} J_{x x}+\theta\left(f_{t}\right) J_{x}, \quad \delta_{2}(\cdot)<\bar{x}<\delta_{1}(\cdot)$,

(3) $\gamma J=x^{2}+\frac{\sigma^{2}}{2} J_{x x}+\theta_{1} J_{x}, \quad J_{x} \geqslant 0, \quad \bar{x} \geqslant \delta_{1}(\cdot)$,

where we assume that whenever $\bar{x}$ finds itself within the tolerance band, $\left.] \delta_{2}(\cdot), \delta_{1}(\cdot)\right]$, the drift of the process is $\theta\left(f_{t}\right)$.

We solve system (10) - details are reported in the Appendix - to derive the threshold values, $\delta_{1}(\cdot)$ and $\delta_{2}(\cdot)$, and the optimal drift, $\theta^{*}\left(f_{t}\right)$, for the process described by (5)

$$
\theta^{*}\left(f_{t}\right)=\left\{\begin{array}{ll}
\theta\left(f_{t}\right) & \delta_{2}<\bar{x}<\delta_{1} \\
\theta_{1} & \bar{x} \geqslant \delta_{1} \\
\theta_{2} & \bar{x} \leqslant \delta_{2}
\end{array} \quad \bar{x}=x_{0}\right.
$$

Solution (11) says that MMAA push as hard as possible to reduce the difference $x_{t}=f_{t}-\tilde{f}_{t}$. This means to move $x_{t}$ to the right of the critical point $\delta_{2}(\cdot)$ when $\bar{x} \leqslant \delta_{2}$ and, vice versa, to the left whenever $\bar{x} \geqslant \delta_{1}(\cdot)$. Given the definition of $x_{t}(4)$, the fundamental dynamics become

$$
\theta^{*}\left(f_{t}\right)= \begin{cases}\theta\left(f_{t}\right), & \tilde{f}_{t}+\delta_{2}(\cdot)<f_{0}<\delta_{1}(\cdot)+\tilde{f}_{t}, \\ \theta_{1}, & f_{0} \geqslant \delta_{1}(\cdot)+\tilde{f}_{t}, \\ \theta_{2}, & f_{0} \leqslant \delta_{2}(\cdot)+\tilde{f}_{t},\end{cases}
$$

where $f_{0}$ is the initial point of the current fundamental.

Expression (12) says that MMAA's aim is to move $f_{t}$ to the right (left) of the critical point $\delta_{2}(\cdot)+\tilde{f}_{t}\left(\delta_{1}(\cdot)+\tilde{f}_{t}\right)$ when $f_{t} \leqslant \delta_{2}(\cdot)+\tilde{f}_{t}\left(f_{t} \geqslant \delta_{1}(\cdot)+\tilde{f}_{t}\right)$. 
We assume that whenever $f_{t}$ exceeds the tolerance band $\left(f_{t} \notin\left[\delta_{2}(\cdot)+\tilde{f}_{t}, \delta_{1}(\cdot)+\tilde{f}_{t}\right]\right)$, some kind of monetary policy interventions occur aimed at bringing $f_{t}$ back into the tolerance band, while no action is taken if $f_{t}$ moves within the tolerance band. The intervention generates an inversion in the trend of the fundamental and causes a switch in regime (state), bringing the process again within the tolerance band according to the SDE (2).

\subsection{The exchange rate dynamics}

In order to express equation (1) in state-space rather than time-series representation, we consider its solution as a continuous twice-differentiable function of the fundamental

$$
s_{t}=h\left(f_{t}\right) .
$$

Applying Ito's lemma we obtain the dynamics of $s$, which is described by ${ }^{2}$

$$
\mathrm{d} s=h^{\prime}(f) \mathrm{d} f+\frac{1}{2} h^{\prime \prime}(f)[\mathrm{d} f]^{2}=h^{\prime}(f)[\theta(f) \mathrm{d} t+\sigma \mathrm{d} B]+\frac{1}{2} h^{\prime \prime}(f) \sigma^{2} \mathrm{~d} t .
$$

Taking the conditional expectation of (14) and using the law of motion (1) we obtain

$$
E_{t} \mathrm{~d} s=h^{\prime} E_{t} \theta(f) \mathrm{d} t+\frac{1}{2} h^{\prime \prime} \sigma^{2} \mathrm{~d} t=\frac{1}{\lambda}(s-f) \mathrm{d} t .
$$

Solving (15) we obtain an expression for the exchange rate related to the fundamental described by (2). ${ }^{3}$ Using Krugman's approach $(1991,1992)$, we obtain the general closed form solution of (15)

$$
s=h(f)= \begin{cases}f+\lambda \theta_{1}+G_{1} e^{v_{1} f}+I_{1} e^{o_{1} f}, & f_{0} \geqslant \delta_{1}(\cdot), \\ f+\lambda \theta_{0}+G_{0} e^{v_{0} f}+I_{0} e^{o_{0} f}, & \delta_{2}(\cdot)<f_{0}<\delta_{1}(\cdot), \\ f+\lambda \theta_{2}+G_{2} e^{v_{2} f}+I_{2} e^{o_{2} f}, & f_{0} \leqslant \delta_{2}(\cdot),\end{cases}
$$

where we assume that within the tolerance band the drift is constant and equal to $\theta_{0}=\theta\left(f_{t}\right)$, and

$$
\begin{aligned}
& v_{i}=\sigma^{-2}\left(-\theta_{i}+\sqrt{\theta_{i}^{2}+2 \frac{\sigma^{2}}{\lambda}}\right)>0 \\
& o_{i}=\sigma^{-2}\left(-\theta_{i}-\sqrt{\theta_{i}^{2}+2 \frac{\sigma^{2}}{\lambda}}\right)<0
\end{aligned} i=0,1,2
$$

In (16) we need to determine $G_{i}(i=0,1,2)$ and $I_{i}(i=0,1,2)$ according to the following boundary conditions

$$
\begin{aligned}
& \text { (1) } G_{0}=0, I_{0}=0, \\
& \text { (2) } G_{1}=0, I_{2}=0, \\
& \text { (3) }\left\{\begin{array}{l}
\lambda \theta_{1}+I_{1} e^{o_{1} \delta_{1}}=\lambda \theta_{0}, \\
\lambda \theta_{2}+I_{2} e^{o_{2} \delta_{2}}=\lambda \theta_{0},
\end{array}\right.
\end{aligned}
$$

where

\footnotetext{
${ }^{2}$ To simplify notations we omit, where possible, time dependence.

${ }^{3}$ To simplify notations we assume, without loss of generality, that the initial level for the theoretical fundamental is $\tilde{f}_{0}=0$.
} 


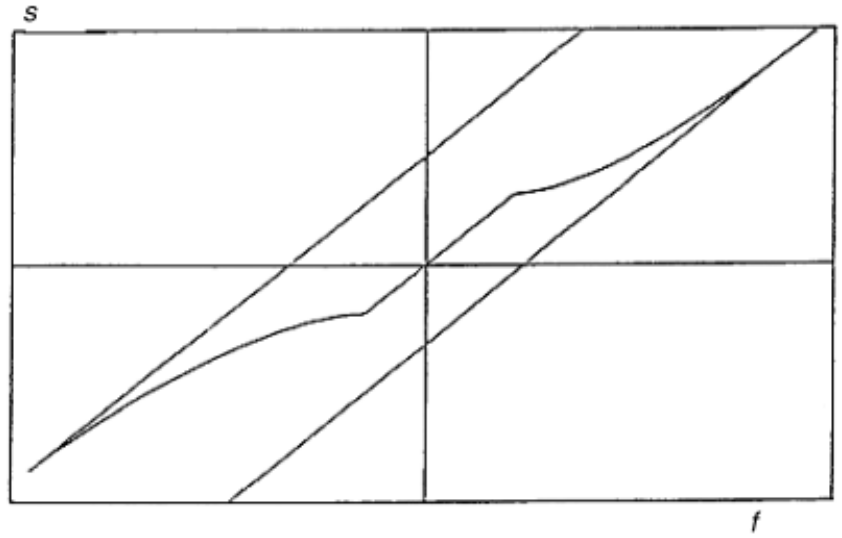

Fig. 1. Exchange rate dynamics within the tolerance band.

- condition (1) implies that inside the tolerance band $] \delta_{2}(\cdot), \delta_{1}(\cdot)[$ MMAA do not adopt any policy measure to modify the dynamics of the fundamental, hence the correct solution is defined by the linear component of (15);

- condition (2) derives from the asymptotic behaviour of $s$ :

$$
\begin{aligned}
& \lim _{f \rightarrow+\infty} h(f)=f+\lambda \theta_{1}, \\
& \lim _{f \rightarrow-\infty} h(f)=f+\lambda \theta_{2},
\end{aligned}
$$

which varies within the band defined by $f+\lambda \theta_{1}$ and $f+\lambda \theta_{2}$;

- condition (3) avoids the occurrence of discrete jumps at $f=\delta_{i}(i=1,2)$.

The final solution, therefore, is defined by

$$
\begin{array}{ll}
s=f+\lambda \theta_{0}, & \delta_{2}<f<\delta_{1}, \\
s=f+\lambda \theta_{1}-\lambda\left(\theta_{1}-\theta_{0}\right) e^{o_{1}\left(f-\delta_{1}\right)}, & f \geqslant \delta_{1}, \\
s=f+\lambda \theta_{2}-\lambda\left(\theta_{2}-\theta_{0}\right) e^{v_{2}\left(f-\delta_{2}\right)}, & f \leqslant \delta_{2} .
\end{array}
$$

Figure 1 describes the dynamics of the exchange rate, $s$, solution of (15).

\section{Model's validation}

In this section we provide a validation of the model described in Section 2. We use monthly data for the D-Mark/US-dollar exchange rate over the period January 1973 (the beginning of the general floating exchange rate system) to January 2005.

To estimate the parameters characterizing the fundamental dynamics we use the simulated moments estimation (SME). The estimated parameters have been statistically tested.

We use the following specification for the monetary model:

$$
s_{t}=f_{t}+\lambda\left[E_{t}\left(s_{t+1}-s_{t}\right)\right] \text {, }
$$


Table 1

OLS results for equation (24)

\begin{tabular}{llll}
\hline$g$ & $t$ & $\sigma_{g}$ & $R^{2}$ \\
\hline 0.8 & 2.28 & 0.054 & 0.67 \\
\hline
\end{tabular}

where $\lambda$ is the semi-elasticity of domestic money demand; $s_{t}$ is the logarithm of the spot exchange rate (in terms of home currency, D-Mark, per unit of foreign currency, US-dollar); $E_{t}$ is the conditional expectations operator defined by $E_{t}\left(s_{t+1}\right) \equiv E_{t}\left(s_{t+1} \mid \Im_{t}\right) ; f_{t}$ defines the fundamental.

We assume the observability of the fundamental (Svensson, 1992). In addition, given the Uncovered Interest Parity holds, ${ }^{4} f_{t}$ is obtained from (21) as the difference between the spot exchange rate, $s_{t}$, and the one-month interest rate differential $\left(r_{t}-r_{t}^{*}\right)$ :

$$
\hat{f}_{t}=s_{t}-\hat{\lambda}\left(r_{t}-r_{t}^{*}\right) \text {. }
$$

According to Huang (1981), a realistic estimation of the fundamental is obtained assuming $\hat{\lambda}=1.5$.

In this model, MMAA's interventions are introduced when the difference $x_{t}=f_{t}-\tilde{f}_{t}$ exceeds the tolerance band $] \delta_{2}(\cdot), \delta_{1}(\cdot)\left[\right.$. The threshold values, $\delta_{1}(\cdot)$ and $\delta_{2}(\cdot)$, and the strength of the control, $\theta\left(f_{t}\right)$, in (5) are determined as the solution of the stochastic control problem described by (8).

We estimate the theoretical level $\tilde{f}_{t}$ using the $\hat{f}_{t}$ described by (22) and assuming the term $\beta_{t}$ in (3) is defined by

$$
\beta_{t}=g\left(r_{t}-r_{t}^{*}\right) .
$$

Standard OLS technique on the difference of $\hat{f}_{t}$ allows the estimation of $g$ :

$$
\Delta \hat{f}_{t}=g\left(\Delta r_{t}-\Delta r_{t}^{*}\right)+\varepsilon_{t} .
$$

The results of the estimation of (24) are reported in Table 1.

\subsection{Estimation of the fundamental}

The process for the fundamental, $f_{t}$, is generated by the difference equation

$$
f_{t}^{\xi}=h\left(f_{t-1} ; \varepsilon_{t} ; \xi_{0}\right),
$$

where $h$ is the transition function; $\xi_{0}=\left(\theta_{1}, \theta_{2}, \theta^{+}, \theta^{-}, \sigma, \gamma\right)$ is the unknown q-dimensional vector and $\left\{\varepsilon_{t}\right\}$ is an i.i.d. sequence of $N(0,1)$ random variables, defined on the probability space $(\Omega, \Im, P)$. To calibrate the model and estimate $\theta^{*}(f t)$ according to (12), we make some realistic assumptions about $\theta(f t)$. Precisely, if the process, $f_{t}$, moves within the tolerance band $\tilde{f}_{t}+\delta_{2}<f_{t}<\delta_{1}+\tilde{f}_{t}$, after the adoption of expansionary (contractionary) policies, the drift of the process $\theta(f t)$ assumes a constant positive (negative) value: $\theta^{+}\left(\theta^{-}\right)$.

From (25) a set of simulated equilibrium states, $\left\{f_{t}^{\xi}\right\}_{t=1}^{N}$, can be generated. For some chosen observation function $h$, in each period $t$ an observation $Z=h\left(f_{t}, f_{t-1}, \ldots, f_{t-l-1}\right)$ is made of a

\footnotetext{
${ }^{4}$ According to the UIP: $E_{t}\left(s_{t+1}-s_{t}\right)=r_{t}-r_{t}^{*}$.

(C) 2007 The Authors.

Journal compilation (C) 2007 International Federation of Operational Research Societies
} 


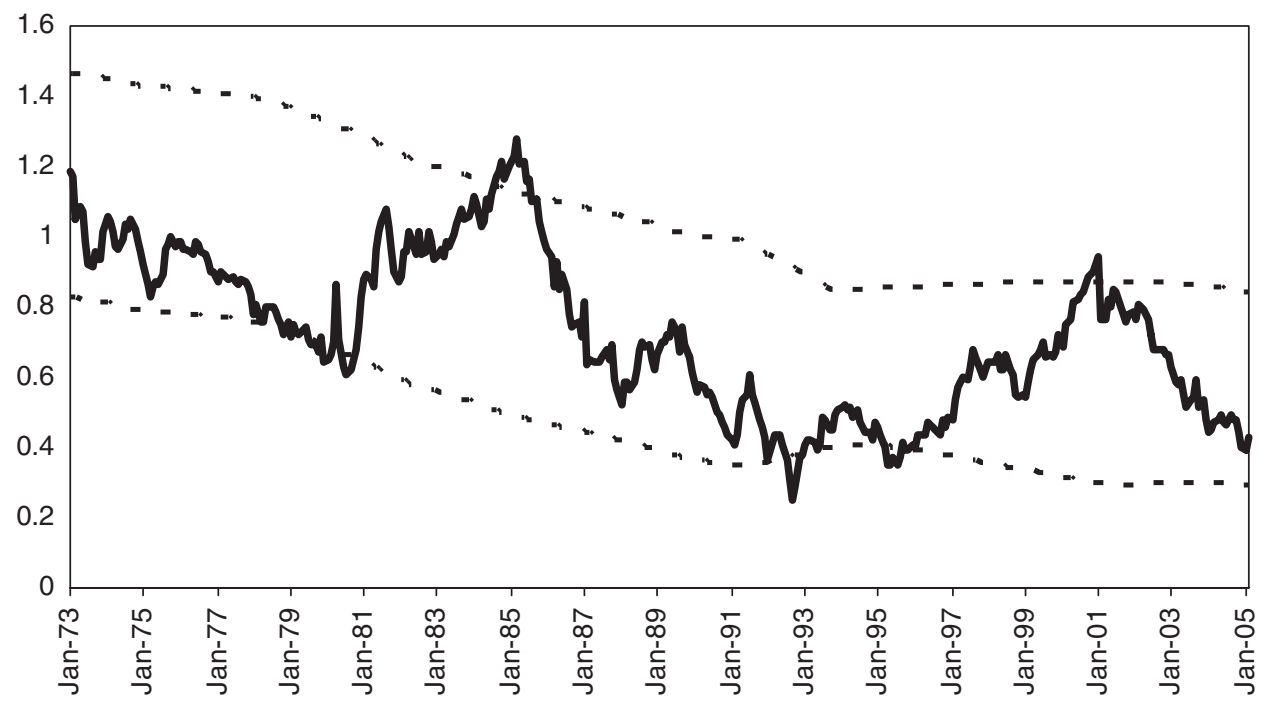

Fig. 2. Fundamental dynamics within and outside the tolerance band.

finite '1-history' of state information. A corresponding observation of $\left\{f_{t}^{\xi}\right\}$ can be formed for each 'l-history' of simulated states. Finally, the SME sets a value of $\xi$ chosen to minimize the distance between the sample first three moments of $\left\{f_{t}^{\xi}\right\}_{t=1}^{N}$ and the sample first three moments of $\left\{\hat{f}_{t}\right\}_{t=1}^{T}$, where $T$ is the number of historical observations (Duffie and Singleton, 1993; Newey, 2001). The same procedure is repeated on the first differences of $f, \Delta f$.

For each initial point $\hat{f}_{0}=f_{0}$ and each parameter vector $\xi \in \Xi$, the simulated state process $\left(\hat{f}_{t}^{\xi}\right)$ is built as

$$
\begin{aligned}
& f_{0}^{\xi}=\hat{f}_{0}, \\
& f_{t+1}^{\xi}=h\left(\hat{f}_{t}^{\xi}, \hat{\varepsilon}_{t+1}, \xi\right) .
\end{aligned}
$$

Using the SME, we compute the first three moments, $\mu_{1}, \mu_{2}, \mu_{3}$ of $\left\{f_{t}^{\xi}\right\}_{t=1}^{N}$, and the first three moments, $\mu_{1}{ }^{\prime}, \mu_{2}{ }^{\prime}, \mu_{3}{ }^{\prime}$ of $\left\{\Delta f_{t}^{\xi}\right\}_{t=1}^{N}$. Hence we have an exactly identified case. We run 1200 simulations and estimate the vector $\xi_{0}=\left(\theta_{1}, \theta_{2}, \theta^{+}, \theta^{-}, \sigma, \gamma\right)$, obtaining

\begin{tabular}{|l|l|l|l|l|l|}
\hline$\hat{\theta}_{1}$ & $\hat{\theta}_{2}$ & $\hat{\theta}^{+}$ & $\hat{\theta}^{-}$ & $\hat{\sigma}$ & $\hat{\gamma}$ \\
\hline-0.0376 & 0.03469 & 0.003114 & -0.00385 & 0.0922 & 0.1185 \\
\hline
\end{tabular}

Solving the system of nonlinear equations (16), we obtain the estimated tolerance band, $\left.\delta_{i}\left(\theta_{j}, \gamma\right),\right]-0.3052 ; 0.43196[$. In Fig. 2 the dynamics for the estimated fundamental within the tolerance band $]-0.30052+\tilde{f}_{t} ; 0.43196+\tilde{f}_{t}[$ is presented.

These results imply:

- if $f_{t}-\tilde{f}_{t} \leqslant-0.3052$, an intervention by MMAA occurs, i.e. expansionary monetary policies generate a positive drift, $\theta_{2}=0.03469$. 
Table 2

Actual and simulated moments: test statistic for the fundamental

\begin{tabular}{lccc}
\hline & Actual & Simulated & $\begin{array}{l}\text { \% of simulated cases } \\
\text { exceeding the } \\
\text { reference ones }\end{array}$ \\
\hline$\mu_{1}(f)$ & 0.824901 & 0.806774 & 48 \\
$\mu_{2}(f)$ & 0.839948 & 0.731886 & 26 \\
$\mu_{3}(f)$ & 0.653658 & 0.720764 & 71 \\
Skewness $(f)$ & -0.14727 & -0.01183 & 22 \\
Kurtosis $(f)$ & -0.71005 & -1.15951 & 89 \\
$\mu_{1}^{\prime}(\Delta f)$ & -0.00054 & -0.00058 & 51 \\
$\mu_{2}^{\prime}(\Delta f)$ & 0.00288 & 0.0007483 & 75 \\
$\mu_{3}^{\prime}(\Delta f)$ & -0.0006 & -0.0026 & 79 \\
Skewness $(\Delta f)$ & -0.12032 & -0.00191 & 6 \\
Kurtosis $(\Delta f)$ & 1.45574 & 0.295208 & 4 \\
$G$ & & 0.0031145 & \\
\hline
\end{tabular}

- if $f_{t}-\tilde{f}_{t} \geqslant 0.43196$, restrictive monetary policies are adopted and the action is reflected in a negative drift for $f_{t}, \theta_{1}=-0.0376$.

- if $-0.3052<f_{t}-f_{t}<0.43196$, MMAA let the fundamental freely float inside the tolerance band. We estimate a positive drift, $\theta_{0}^{+}=0.003114$, and a negative one, $\theta_{0}^{-}=0.00385$, for the appreciating and depreciating regimes, respectively.

The implementation of SME is based on a number of sample moments exactly equal to the number of parameters to be estimated; hence we could not test the estimated model using the over-identifying restriction. As an alternative, we adopt Smith and Spencer's method (1992) and generate $n$ replications using the estimated parameters. In each replication $\mu_{1}, \mu_{2}, \mu_{3}$ and $\mu_{1}{ }^{\prime}, \mu_{2}{ }^{\prime}$, $\mu_{3}{ }^{\prime}$ were estimated, together with a test for normality. The results are reported in Table 2, where the sets of the first three moments on the levels and on the first differences of the observed and the simulated series are listed. On each of these series the statistics for skewness and kurtosis are reported. The moments for the simulated series were obtained using $n=100$ replications.

In the last column of Table 2 the percentage of replications in which the simulated value is greater than the observed one, for positive sample statistics, or is less than the observed value, for negative ones, is also reported. On average, 51 out of 100 replications provide a mean change in the fundamental lower than the change in the observed series. ${ }^{5}$ All the values in Table 2 support the statistical significance of the estimated parameters. The only non-significant statistic refers to the kurtosis of the first differences, showing that the model does not succeed in describing the observed kurtosis.

\footnotetext{
${ }^{5} \mathrm{~A}$ statistic is considered well matched if the proportion of replications in which the simulated value exceeds the historical value falls roughly within the interval $(0.05,0.95)$.
} 


\section{Conclusions}

The paper presents a new theoretical approach to describe the long-swinging behavior of exchange rates observed over the last 30 years.

The dynamics of the exchange rates are described as a function of the macroeconomic fundamental, $f_{t}$, which follows a Brownian motion with state-dependent drift. The process for the dynamics of the fundamental is obtained as the solution of a stochastic control problem, which describes the MMAA's aim to keep the value of the fundamental as close as possible to a predefined long-run path. The optimal control approach allows one to set an endogenous timevarying tolerance band, outside of which an optimal time-consistent policy takes place.

The approach is realistic because it suggests a more adequate process to describe exchange rate dynamics and provides an accurate analysis of the observed phenomenon with respect to simple diffusion processes or MSMs, which may lack economic explanation. The model takes into account MMAA's role in preventing the exchange rate to be set at wrong levels and shows that a change in monetary policy may lead to the long-swinging behavior of exchange rates. Furthermore, we contribute to the re-examination of the monetary model in a time-varying context and to the definition of possible interactions between the exchange rates and the fundamental.

An application of the model is also provided. The estimation of the parameters that define the dynamics of the fundamental supports the optimal control approach and the long-swinging behavior of the exchange rates. The model succeeds in capturing all the major switches in regime observed in the D-Mark/US-dollar exchange rate over the period 1973-2005.

\section{References}

Bollen, N.P.B., Gray, S.F., Whaley, R.E., 2000. Regime switching in foreign exchange rates: evidence from currency option prices. Journal of Econometrics 94, 239-276.

Cheung, Y.W., Chinn, M., Pascual, G.A., 2005. Empirical exchange rate models of the nineties: are any fit to survive? Journal of International Money and Finance 24, 1150-1175.

Cheung, Y.W., Erlandsson, U.G., 2005. Exchange rate and Markov switching dynamic. Journal of Business and Economic Statistics 23, 3, 314-320.

Chinn, M., Meese, R., 1995. Banking on currency forecasts: how predictable is change in money? Journal of International Economics 38, 1-2, 161-178.

Clarida R. H., Sarno, L., Taylor, M.P., Valente, G., 2003. The out-of-sample success of term structure models as exchange rate predictors: a step beyond. Journal of International Economics 60, 1, 61-83.

Dewachter, H., 2001. Can Markov switching models forecast exchange rates? Journal of International Economics $36,151-165$.

Duffie, D., Singleton, K.J., 1993. Simulated moments estimation of Markov models of asset prices. Econometrica 61, 929-952.

Engel, C., 1994. Can the Markov switching model forecast exchange rates? Journal of International Economics 36, 151165.

Engel, C., Hamilton, J.D., 1990. Long swings in the dollar: are they in the data and do markets know it? The American Economic Review 4, 689-711.

Evans, M., Lewis, K.K., 1995. Do long-term swings in the dollar affect estimates of the risk premia? Review of Financial Studies 8, 709-742. 
Huang, R.D., 1981. The monetary approach to exchange rate in an efficient foreign exchange market: tests based on volatility. The Journal of Finance 1, 31-41.

Kaminsky, G., 1993. Is there a peso problem? Evidence from the Dollar/Pound exchange rate, 1976-1987. American Economic Review 83, 450-472.

Krugman, P., 1991. Target zones and exchange rate dynamics. Quarterly Journal of Economics 56, 669-682.

Krugman, P., 1992. Exchange Rate in a Currency Band: A Sketch of the New Approach. Cambridge University Press, Cambridge, UK, pp. 9-14.

MacDonald, R., 1999. Exchange rate behaviour: are fundamentals important? Economic Journal 109, 459, 673-691.

MacDonald, R., Taylor, M.P., 1994. The monetary approach to the exchange rate: long-run relationships, short run dynamics and how to beat a random walk. Journal of International Money and Finance 13, 3, 267-276.

Mark, N., 1995. Exchange rates and fundamentals: evidence on long-horizon predictability. American Economic Review $85,1,201-218$.

Marsh, I.W., 2000. High frequency Markov switching models in the foreign exchange market. Journal of Forecasting 19, $123-134$.

Meese, R., Rogoff, K., 1983. Empirical exchange rate models of the seventies: do they fit out of sample? Journal of International Economics 14, 3-24.

Meese, R., Rose, A.K., 1991. An empirical assessment in models of exchange rate determinations. Review of Economic Studies 80, 608-619.

Miller, M., Zhang, L., 1996. Optimal target zones: how an exchange rate mechanism can improve upon discretion. Journal of Economic Dynamic and Control 20, 1641-1660.

Mundaca, G., 2001. Central bank interventions and exchange rate band regimes. Journal of International Financial Markets, Institutions and Money 20, 677-700.

Mussa, M., 1981. The role of official intervention. Group of Thirty Occasional Paper No. 6, New York, Group of Thirty.

Newey, W.K., 2001. Flexible simulated moment estimation of non-linear errors in variable models. Review of Economics and Statistics 83, 4, 616-627.

Smith, G.W., Spencer, M.G., 1992. Estimation and testing in models of exchange rate target zones and process switching. In: Krugman, P., Miller, M. (Eds.), Exchange Rate Targets and Currency Bands. Cambridge University Press, Cambridge, UK.

Svensson, L.E.O., 1992. An interpretation of recent research on exchange rate target zones. Journal of Economic Perspectives 6, 119-144.

\section{Appendix A}

We look for solutions $J=O\left(x^{2}\right)$ as $|x| \uparrow \infty$ of (10) which meet smoothly at $x=\delta_{2}$ and $x=\delta_{1}$ to order 1 , since the cost of control increases nonlinearly when $x_{t}$ is far from the origin. As a particular solution of the first equation in (10) we find

$$
\frac{x^{2}}{\gamma}+\frac{\sigma^{2}}{\gamma^{2}}+\frac{2 \theta_{2} x}{\gamma^{2}}+\frac{2 \theta_{2}^{2}}{\gamma^{3}} \quad \bar{x} \leqslant \delta_{2} .
$$

A general solution of (10) is obtained adding to (A.1) a solution of $\gamma J=\frac{\sigma^{2}}{2} J_{x x}+\theta_{2} J_{x}$ given by

$$
C_{2} e^{\lambda_{2} x}+D_{2} e^{\eta_{2} x}
$$

where

(C) 2007 The Authors.

Journal compilation (C) 2007 International Federation of Operational Research Societies 


$$
\begin{aligned}
& \lambda_{2}=\sigma^{-2}\left(-\theta_{2}+\sqrt{\theta_{2}^{2}+2 \gamma \sigma^{2}}\right)>0, \\
& \eta_{2}=\sigma^{-2}\left(-\theta_{2}-\sqrt{\theta_{2}^{2}+2 \gamma \sigma^{2}}\right)<0,
\end{aligned}
$$

and $C_{2}, D_{2}$ are arbitrary constants.

A similar approach is applied for solutions of the other two equations in (10), so the general solution is

$$
\gamma J= \begin{cases}\frac{x^{2}}{\gamma}+\frac{\sigma^{2}}{\gamma^{2}}+\frac{2 \theta_{2} x}{\gamma^{2}}+\frac{2 \theta_{2}^{2}}{\gamma^{3}}+C_{2} e^{\lambda_{2} x}+D_{2} e^{\eta_{2} x}, & \bar{x} \leqslant \delta_{2}, \\ \frac{x^{2}}{\gamma}+\frac{\sigma^{2}}{\gamma^{2}}+\frac{2 \theta_{0} x}{\gamma^{2}}+\frac{2 \theta_{0}^{2}}{\gamma^{3}}+C_{0} e^{\lambda_{0} x}+D_{0} e^{\eta_{0} x}, & \delta_{2}<\bar{x}<\delta_{1}, \\ \frac{x^{2}}{\gamma}+\frac{\sigma^{2}}{\gamma^{2}}+\frac{2 \theta_{1} x}{\gamma^{2}}+\frac{2 \theta_{1}^{2}}{\gamma^{3}}+C_{1} e^{\lambda_{1} x}+D_{1} e^{\eta_{1} x}, & \bar{x} \leqslant \delta_{1},\end{cases}
$$

where terms $\lambda_{i}$ and $\eta_{i}$ for $(i=0,1,2)$ are defined as in (A.3), using the corresponding drifts $\theta_{j}$, $j=0,1,2$. The final expression for the threshold values $\delta_{1}\left(\theta_{j}, \gamma\right)$ and $\delta_{2}\left(\theta_{j}, \gamma\right)$ is obtained solving (A.4) and defining the six arbitrary constants, $C_{i}(i=0,1,2)$ and $D_{i}(i=0,1,2)$.

We are able to obtain the values for $\delta_{1}$ and $\delta_{2}$ as $f\left(\theta_{0}, \theta_{1}, \theta_{2}, \gamma\right)$ solving a system of nonlinear equations

$$
\left\{\begin{array}{l}
a_{11} e^{\lambda_{0} \delta_{2}-\lambda_{0} \delta_{1}}+a_{12} e^{\eta_{2} \delta_{2}-\eta_{0} \delta_{1}}=b_{11}, \\
a_{21} e^{\lambda_{0} \delta_{2}-\lambda_{0} \delta_{1}}+a_{22} e^{\eta_{2} \delta_{2}-\eta_{0} \delta_{1}}=b_{21},
\end{array}\right.
$$

Equation (A.5) is obtained substituting the value of the constants $C_{0}, C_{1}, C_{2}, D_{0}, D_{1}, D_{2}$ in expression (A.4).

$C_{i}(i=0,1,2)$ and $D_{i}(i=0,1,2)$ are obtained imposing the quadratic growth condition; for $|x| \uparrow \infty, C_{2}=0$ and $D_{1}=0$. The other four arbitrary constants are obtained assuming continuity of $J(x)$ and its first derivative, $J_{x}(x)$, in $x=\delta_{2}$ and $x=\delta_{1}$, so we get the following four equations to determine $C_{1}, C_{0}, D_{0}$, and $D_{2}$.

$$
\left\{\begin{array}{l}
\frac{2 \theta_{0}}{\gamma^{2}}-\frac{2 \theta_{1}}{\gamma^{2}}+C_{0} \lambda_{0} e^{\lambda_{0} \delta_{1}}-C_{1} \lambda_{1} e^{\lambda_{1} \delta_{1}}+D_{0} \eta_{0} e^{\eta_{0} \delta_{1}}=0, \\
\frac{2\left(\theta_{0}-\theta_{1}\right)}{\gamma^{2}} \delta_{1}+\frac{2 \theta_{0}^{2}}{\gamma^{3}}-\frac{2 \theta_{1}^{2}}{\gamma^{3}}+C_{0} e^{\lambda_{0} \delta_{1}}-C_{1} e^{\lambda_{1} \delta_{1}}+D_{0} \eta_{0} e^{\eta_{0} \delta_{1}}=0, \\
\frac{2 \theta_{0}}{\gamma^{2}}-\frac{2 \theta_{2}}{\gamma^{2}}+C_{0} \lambda_{0} e^{\lambda_{0} \delta_{2}}-D_{2} \eta_{2} e^{\eta_{2} \delta_{2}}+D_{0} \eta_{0} e^{\eta_{0} \delta_{2}}=0, \\
\frac{2\left(\theta_{0}-\theta_{2}\right)}{\gamma^{2}} \delta_{2}+\frac{2 \theta_{0}^{2}}{\gamma^{3}}-\frac{2 \theta_{2}^{2}}{\gamma^{3}}+C_{0} e^{\lambda_{0} \delta_{2}}-D_{2} e^{\eta_{2} \delta_{2}}+D_{0} e^{\eta_{0} \delta_{2}}=0 .
\end{array}\right.
$$

Solving system (A.6) and assuming also continuity of the second derivative $J_{x x}(x)$ for $x=\delta_{2}$ and $x=\delta_{1}$, we get an expression for $C_{1}$ and $D_{2}$ as function of $C_{0}$ and $D_{0}$

$$
\left\{\begin{array}{l}
C_{1} \lambda_{1} e^{\lambda_{1} \delta_{1}}=D_{0} \eta_{0}^{2} e^{\eta_{0} \delta_{1}}+C_{0} \lambda_{0}^{2} e^{\lambda_{0} \delta_{1}} \\
D_{2} \eta_{2}^{2} e^{\eta_{2} \delta_{2}}=D_{0} \eta_{0}^{2} e^{\eta_{0} \delta_{2}}+C_{0} \lambda_{0}^{2} e^{\lambda_{0} \delta_{2}}
\end{array}\right.
$$


Substituting (A.7) in (A.6), after some algebra, we get the final expressions for $C_{0}, C_{1}, D_{0}$ and $D_{2}$

$$
\begin{aligned}
& C_{0}=\frac{p_{1} \lambda_{1} \eta_{0}-q_{1}\left(\lambda_{1}-\eta_{0}\right)}{\left(\eta_{0}-\lambda_{0}\right)\left(\lambda_{1}-\lambda_{0}\right)} e^{-\lambda_{0} \delta_{1}}, \\
& C_{1}=\frac{C_{0} \lambda_{0}^{2} e^{\left(\lambda_{0}-\eta_{2}\right) \delta_{2}}+D_{0} \eta_{0}^{2} e^{\left(\eta_{0}-\eta_{2}\right) \delta_{2}}}{\eta_{2}^{2}}, \\
& D_{0}=\frac{p_{1} \lambda_{1} \lambda_{0}-q_{1}\left(\lambda_{1}+\lambda_{0}\right)}{\left(\lambda_{0}-\eta_{0}\right)\left(\eta_{1}-\eta_{0}\right)} e^{-\eta_{0} \delta_{1}}, \\
& D_{2}=\frac{C_{0} \lambda_{0}^{2} e^{\left(\lambda_{0}-\lambda_{1}\right) \delta_{1}}+D_{0} \eta_{0}^{2} e^{\left(\eta_{0}-\lambda_{1}\right) \delta_{1}}}{\lambda_{1}^{2}},
\end{aligned}
$$

with

$$
\begin{aligned}
& q_{1}=\frac{2}{\gamma^{2}}\left(\theta_{0}-\theta_{1}\right), \\
& q_{2}=\frac{2}{\gamma^{2}}\left(\theta_{0}-\theta_{2}\right), \\
& p_{1}=\delta_{1} q_{1}+\frac{2}{\gamma^{3}}\left(\theta_{0}^{2}-\theta_{1}^{2}\right), \\
& p_{2}=\delta_{2} q_{2}+\frac{2}{\gamma^{3}}\left(\theta_{2}^{2}-\theta_{0}^{2}\right) .
\end{aligned}
$$

Furthermore, it could be verified that non-negative constants $C_{1}$ and $D_{2}$ guarantee the convexity of function $J(x)$.

Once defined the arbitrary constants $C_{0}, C_{1}, D_{0}$ and $D_{2}$, in order to get the expression for $\delta_{2}$ and $\delta_{1}$ as $f\left(\theta_{0}, \theta_{1}, \theta_{2}, \gamma\right)$, we impose the values of the solutions for $J(x)$ to match at $x=\delta_{2}$ and $x=\delta_{1}$, and we get the following system of nonlinear equations

$$
\left\{\begin{array}{l}
a_{11} e^{\lambda_{0} \delta_{2}-\lambda_{0} \delta_{1}}+a_{12} e^{\eta_{2} \delta_{2}-\eta_{0} \delta_{1}}=b_{11}, \\
a_{21} e^{\lambda_{0} \delta_{2}-\lambda_{0} \delta_{1}}+a_{22} e^{\eta_{2} \delta_{2}-\eta_{0} \delta_{1}}=b_{21},
\end{array}\right.
$$

with

$$
\begin{aligned}
& a_{11}=l\left[q_{2}\left(\lambda_{0}+\eta_{2}\right)\right], \\
& a_{12}=j\left[p_{2} \eta_{2}^{2}-\left(\eta_{0}+\eta_{2}\right)\left(q_{2}-\eta_{2} p_{2}\right)\right], \\
& a_{21}=l\left[-q_{1}\left(\lambda_{1}+\eta_{0}\right)+\lambda_{1} \eta_{0} p_{1}\right], \\
& a_{22}=j\left[-q_{1}\left(\lambda_{0}+\eta_{2}\right)+\lambda_{0} \lambda_{1} p_{1}\right], \\
& b_{11}=k\left[q_{1}-\lambda_{0} p_{1}\right], \\
& b_{21}=m\left[\left(q_{2}-\eta_{2} p_{2}\right)\left(\lambda_{0}+2 \lambda_{1}+\eta_{0}\right)\right],
\end{aligned}
$$


where

$$
\begin{gathered}
j=\left(\eta_{0}-\eta_{2}\right)\left(\lambda_{0}-\lambda_{1}\right), \\
k=\left(\lambda_{1}-\eta_{0}\right)\left(\lambda_{0}-\lambda_{1}\right), \\
l=\left(\lambda_{1}-\eta_{0}\right)\left(\lambda_{0}-\eta_{2}\right), \\
m=\left(\eta_{0}-\eta_{2}\right)\left(\lambda_{0}-\eta_{2}\right),
\end{gathered}
$$

which is solved using Newton's algorithm. 